\title{
The Drawing of Configurations
}

\author{
Harald Gropp \\ Mühlingstr.19, D-69121 Heidelberg, Germany
}

\begin{abstract}
The drawing of configurations and other linear hypergraphs is discussed. From their historical and geometrical context it is quite natural to denote hyperedges of vertices as lines, i.e. to position the points (vertices) in the plane such that those points which form hyperedges are collinear in the plane (or as close to collinear as possible). This is a new concept in the area of hypergraph drawing. However, in mathematics it has been used for more than 100 years. The exact drawing of configurations is mainly based on the realization of matroids and techniques in computer algebra.
\end{abstract}

\section{Introduction and Notation}

\subsection{Graphs andGeometry}

The topic of graph drawing is interesting not only from the point of view of finding nice drawings of graphs for all kinds of applications. It is, of course, the fascinating meeting of two mathematical theories, geometry and graph theory.

Geometry is one of the oldest mathematical theories which reached a high level already more than 2000 years ago. It was and perhaps is still dominated by the axiomatic way of Euclid. The appearance of noneuclidean geometries in the $19^{\text {th }}$ century extended the meaning of geometry within mathematics. However, in graph drawing the main task is still to display graphs in 2-dimensional ( or 3-dimensional ) geometry.

The development of graph theory started from many different applications in a rather different way. Many important roots can be found in sciences like physics and chemistry in the $19^{\text {th }}$ century whereas graph theory was established as a theory of its own around 1960 .

This paper being in the section Graphs defined by geometry discusses the drawing of hypergraphs in general and configurations in particular. Whether these combinatorial structures are regarded to be defined by geometry or whether they define new kinds of geometry ( finite and noneuclidean) depends on the point of view.

These "geometric" structures contain only a finite number of points ( and hence lines ). It may even occur that there is no line connecting two points which clearly contradicts to the axioms of Euclid. This makes it possible that many new geometric structures arise which are "counterexamples" to classical or more modern theorems of euclidean geometry (Pappus or Sylvester-Gallai ).

The problem of drawing linear hypergraphs is to obtain ( of course) nice drawings of those structures which exist in euclidean geometry and to produce 
reasonable drawings of the "new" structures although they have "noneuclidean" properties.

\subsection{Some Definitions}

Definition 1.1 $A$ hypergraph $H=(V, E)$ consists of a set of vertices $V$ and a set of hyperedges $H$ where each hyperedge is a non-empty subset of $V$. A hypergraph is called $k$-uniform if the size of each hyperedge is $k, r$-regular if each vertex belongs to exactly $r$ hyperedges, and linear if the intersection of two different hyperedges is at most one vertex.

For further information on hypergraphs, see e.g. [1].

Definition 1.2 $A$ configuration $\left(v_{r}, b_{k}\right)$ is a finite incidence structure with $v$ points and $b$ lines such that

(1) there are $k$ points on each line and $r$ lines through each point, and

(2) two different points are connected by a line at most once.

A symmetric configuration $\left(v_{k}, v_{k}\right)$ is shortly denoted by $v_{k}$.

From the point of view of hypergraph theory it is easy to describe configurations as follows.

Remark 1 A configuration $\left(v_{r}, b_{k}\right)$ is a linear $r$-regular $k$-uniform hypergraph with $v$ vertices and $b$ hyperedges.

\subsection{Hypergraph Drawing}

In the business of graph drawing the drawing of hypergraphs plays a specific role. For example, a 3-uniform hypergraph with $v$ vertices has subsets of size 3 as edges. Perhaps the smallest very interesting hypergraph is shown in Fig.1. It has 7 vertices and 7 hyperedges (the 6 lines and the "curved" line).

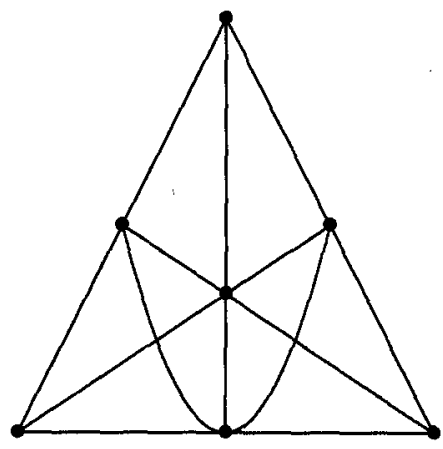

Fig.1.The unique configuration $7_{3}$ 
The usual drawing of edges ( of size 2 ) in a graph is the (linear) segment between its two vertices. The main question is how to position the vertices (as points in the plane or on a grid etc. ) in order to obtain a more or less nice drawing. It depends on the special purpose of the drawing whether the focus is on aspects of symmetry or readability for example.

In the case of hypergraphs the problem becomes much more difficult since through 3 or more given points there is no line (in general). There is a certain class of hypergraphs with a history of more than 100 years, the configurations $\left(v_{r}, b_{k}\right)$. Even their drawing problem was already attacked more than 100 years ago. It should be mentioned that the role of configurations as hypergraphs is just one interesting relation of configurations and graphs (see [5]).

\subsection{Configurations and Their History}

Hence the question arises: If configurations can be described as particular hypergraphs so easily why is there a special name for them? The answer is quite easy: Configurations are much older than hypergraphs. While the term of a hypergraph was introduced around 1960 the first definition of configurations was already given in 1876 in the book Geometrie der Lage of T. Reye at a time when even graph theory had not yet found an established place within mathematics.

It is not only true that configurations are older than graphs and hypergraphs. Thus certain graph-theoretic results were already obtained earlier in the language of configurations. It is also this geometric language of configurations which raises the question of drawability rather naturally. The objects of configurations are called points and lines. However, the language of graph theory uses more abstract terms like vertex and edge. Moreover, the roots of graph theory are very different ones, from logic and topology to physics and chemistry.

Configurations were "born" in geometry. The paragraph where Reye defines configurations is illustrated by a drawing of the famous configuration of Desargues ( see Fig. 2, not identical with the drawing in Reye's book ). By the way, a picture of the Desargues configuration is shown on the cover of the Proceedings of Graph Drawing '93 [3] edited by the Centre d'analyse et de mathématique sociales. 


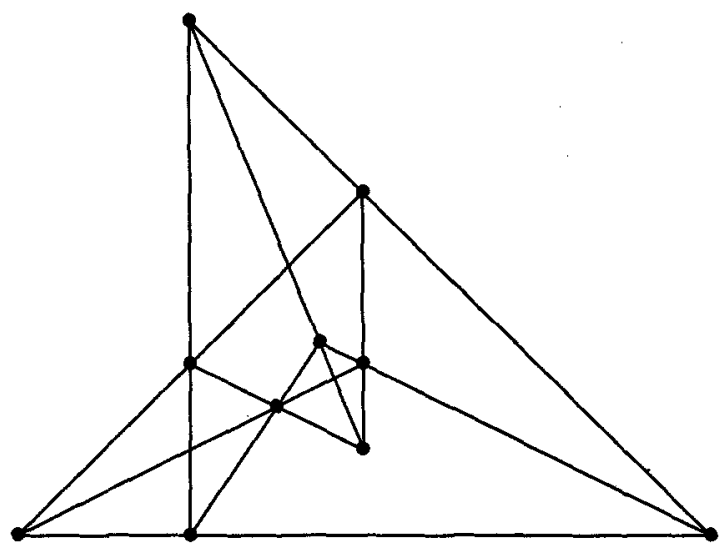

Fig.2.The Desargues configuration $10_{3}$

In the beginning, only those configurations which were drawn in the plane were accepted as "geometric" configurations, the others were called "schematic". Quite soon, however, configurations were regarded as combinatorial structures defined by the axioms given above. It turned out that the configuration $7_{3}$ (the hypergraph of Fig. 1 ) cannot be drawn with straight lines only. Since all its lines contain 3 points such a drawing would be a contradiction to the theorem of Gallai (which was, however, proved later ). In particular, many configurations $v_{3}$ with $v \leq 12$ were constructed (combinatorially) and also drawn as points and lines in the plane. Not all the drawings were correct (compare Fig. 3 below).

A very remarkable result is due E. Steinitz. In his dissertation of 1894 [13] he proved that there is always a drawing for a configuration $v_{3}$ with straight lines and at most one "quadratic" line ( compare Fig. 1).

It is not possible here to describe the history of configurations and their drawings in detail. The interested reader is referred to [4] and [8] for further information. However, I hope to have clarified why the drawing of configurations is not only an interesting topic itself. I think it plays a key role in the drawing of hypergraphs and similar combinatorial structures, because of its long tradition and its geometrical context.

\section{The Different Goals of Drawing}

As already mentioned, it is quite clear that in the drawing of graphs the edges should be realized by straight lines or curves like segments of lines, circles etc. Of course, there remains a lot of discussion how to position the vertices in order to obtain a good, nice, readable etc. drawing of the graph.

In the case of hypergraphs the main strategy until now has been to denote the hyperedge by enclosing the corresponding vertices by a circle or ellipse like curve. The reason is probably that the main root of hypergraph theory is the 
theory of subsets of sets. For these mathematical structures a Venn diagram is the usual tool of drawing pictures.

There is not much literature on drawing of hypergraphs available. The only software for drawing hypergraphs which I have seen until now is the Hypergraph Drawing and Optimization System (HDOS) of Vitaly Voloshin of the State University of Moldova [15]. This HDOS draws hypergraphs as indicated above (Venn like diagrams).

However, since graphs are also hypergraphs, it provides nice drawings of graphs, e.g. of the Petersen graph. Since the Petersen graph has at least 3 nice drawings it is a challenging task for every graph drawing software system. Moreover, the HDOS also computes a lot of graph-theoretical parameters, e.g. the new upper chromatic number, see [14].

In this paper the strategy of drawing will be to describe hyperedges by (straight) lines, at least as far as possible. If the hypergraph is linear, i.e. two hyperedges never intersect in more than one vertex, this aim can be reached perhaps. The result of Steinitz shows that at least for configurations $n_{3}$ it can nearly be reached. Of course, for non-linear hypergraphs the strategy can be bad since in euclidean geometry there is only one line through 2 given points.

\subsection{Realizations over the Real or Rational Numbers}

While the mathematicians of the last century were mainly interested in obtaining nice pictures of their configurations, quite recently in the theory of matroids the question of realizability has been discussed from a more theoretical point of view. By generalizing the concept of independence in linear algebra it was asked whether a given system of subsets can be realized by a matrix such that dependent sets correspond to matrices of determinant 0 . For the following purpose it is not necessary to define matroids here explicitly. For further details on matroids, see e.g. [11].

The problem can be explained in the language of matrices as follows. Given a matrix with 3 rows and $n$ columns with entries in a certain field (e.g. $\mathbb{R}$ ). Identify the set $\{1, \ldots, n\}$ with the set of $n$ columns of the matrix. Determine all 3-by-3 subdeterminants of the matrix. If this determinant is 0 call the set of 3 numbers corresponding to the chosen columns to be a line or hyperedge, otherwise not. This is an algebraic definition of a combinatorial (or geometric) structure.

Vice versa, this problem is closely related to the drawing of hypergraphs. For example, for configurations $v_{3}$ the problem of realization can be defined as follows.

Definition $2.1 A$ coordinatization or realization of a configuration $v_{3}$ with point set $P$ and line set $L$ over a field $K$ is a mapping from $P$ to $K^{3}$ such that for all distinct $i, j, k \in P \operatorname{det}\left(x_{i}, x_{j}, x_{k}\right)=0$ if and only if $i, j, k$ are collinear in the configuration.

For our purposes the field will be the field of real or rational numbers. For details and references concerning this realization problem the reader is referred 
to [8]. As an example the following configuration $11_{3}$ and their realization matrix are given.

Its configuration lines are:

$1,2,4 ; 2,3,5 ; 3,4,6 ; 4,5,7 ; 5,6,8 ; 6,7,9 ; \quad 7,8,10 ; 8,9,11 ; 1,9,10 ; 2,10,11 ; 1,3,11$.

The 3-by-11 matrix

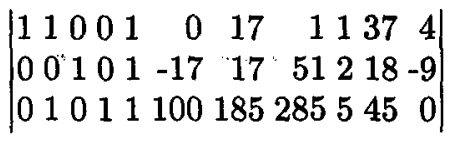

has the property that a 3 -by-3 subdeterminant with columns $i, j, k$ is 0 if and only if $i, j, k$ is one of the 11 lines given above.

In order to obtain a drawing in the 2-dimensional plane a suitable projection of $\mathbb{R}^{3}$ to $\mathbb{R}^{2}$ has to be chosen. What suitable means depends on the further properties of the drawing which we require.

\subsection{Linear Drawings}

At first, it has to be checked whether a configuration can be realized over the real numbers. These realizations have been constructed for all configurations $v_{3}$ with $v \leq 12$ for which this is possible. There are exactly 3 of them which are not realizable over $\mathbb{R}$, the unique configuration $7_{3}$, the unique configuration $8_{3}$, and one configuration $10_{3}$ ( see Fig. 3 ).

E.g. realizations of 8 of the other 9 configurations $10_{3}$ are published in [2]. The Desargues configuration is omitted since its drawing does not cause any problems. The Theorem of Desargues tells us how to draw it, at least on an "infinite" part of paper.

For the convenience of the reader these 8 matrices are printed below. Of course, drawings of these configurations would be nicer, but these matrices are the basis of the drawings and shorten the length of this paper.

$$
\begin{aligned}
& \left|\begin{array}{rrrrrrrrrr}
0 & 12 & 4 & 1 & -1 & -4 & 17 & 0 & 0 & 1 \\
-3 & 6 & -1 & -1 & -2 & 1 & 0 & 1 & 0 & -1 \\
4 & -9 & 1 & 0 & 3 & 3 & 0 & 0 & 1 & 1
\end{array}\right| \\
& \left|\begin{array}{rrrrrrrrrr}
-552 & 48 & -3600 & 2 & 1 & 8 & 0 & 0 & 0 & 1 \\
529 & -42 & 3450 & 4 & 0 & -7 & 4 & 1 & 0 & -1 \\
0 & 117 & -3450 & 71 & 0 & 8 & 71 & 0 & 1 & 1
\end{array}\right|
\end{aligned}
$$

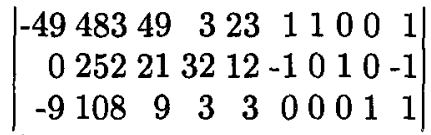

$$
\begin{aligned}
& \left|\begin{array}{rrrrrrrrrr}
-10 & 12 & 0 & 10 & 1 & 18 & 0 & 2 & 0 & 1 \\
-6 & 36 & 36 & 6 & 0 & 18 & 1 & -2 & 0 & -1 \\
6 & 0 & 9 & 9 & 0 & 27 & 0 & 3 & 1 & 1
\end{array}\right|
\end{aligned}
$$




$$
\begin{aligned}
& \left|\begin{array}{rrrrrrrrrr}
89 & -4 & 0 & 4 & 1 & 1 & 0 & 1 & 0 & 1 \\
7832 & 11 & 88 & 85 & 88 & 0 & 1 & -1 & 0 & -1 \\
979 & -11 & 8 & 11 & 8 & 0 & 0 & 0 & 1 & 1
\end{array}\right|
\end{aligned}
$$

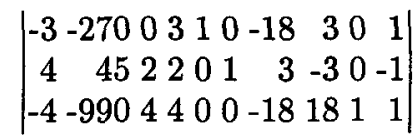

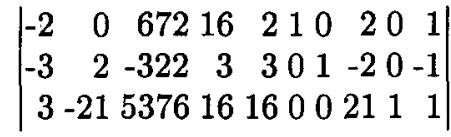

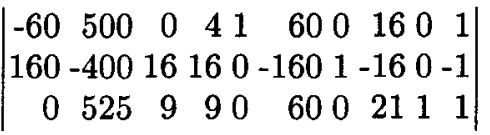

Afterwards a projection yields the required picture. The list of further properties which the drawing should have is quite similar to those for graphs like a certain minimum distance of points, minimum angle between lines, good distribution of points in the picture etc.

\subsection{Approximative Drawings}

From a more practical point of view it is not that bad if a configuration cannot be realized over the reals. Mathematically, this is a remarkable result but it should not discourage us. If the main task of the drawing is to show clearly what exactly the hyperedges or lines are an approximative drawing is suitable as well.

Figure 3 was produced in a paper of S. Kantor of 1881 [10] where he published drawings of all 10 configurations $10_{3}$. However, it was proved a few years later by $\mathrm{E}$. Schroeter and after that several times by other mathematicians that the configuration $10_{3}$ in Fig. 3 is the only one which is not realizable over the reals nor over any other field. In fact, the given drawing is not correct. It is not clear whether Kantor himself knew about it. 


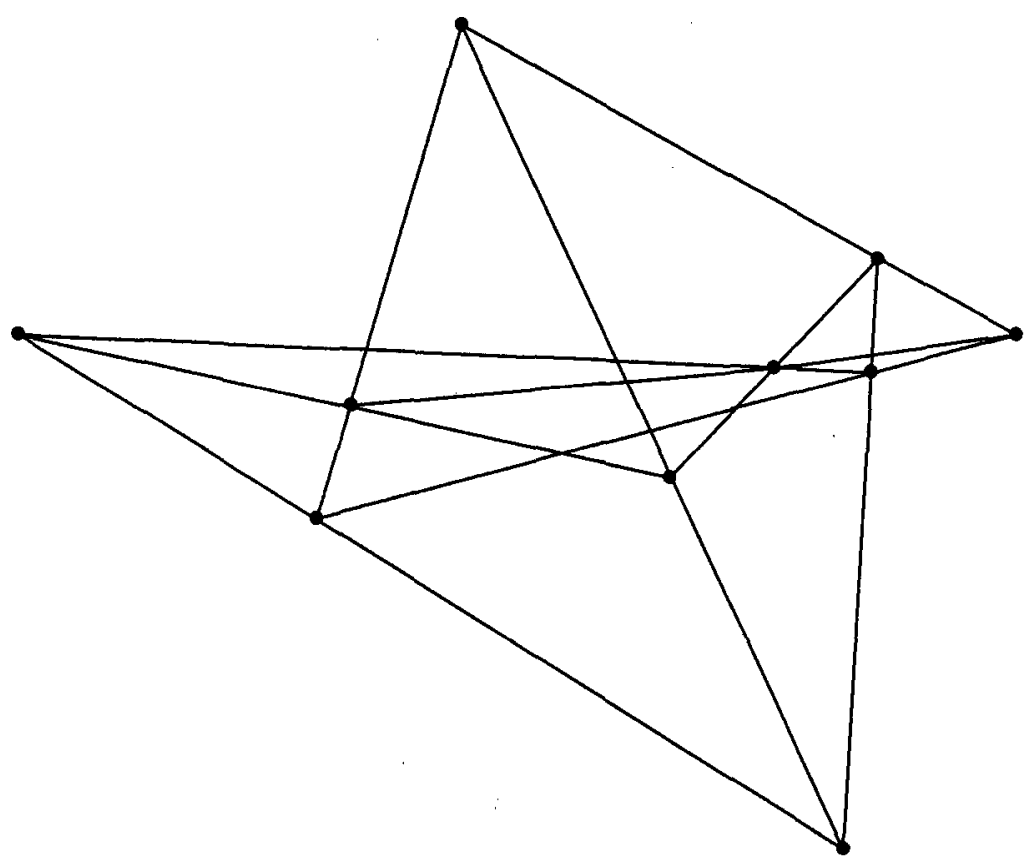

Fig.3.The non-realizable cfz. $10_{3}$

But who cares? It is not so easy to find out which of these 10 "lines" is no line at all. More important everybody will find this drawing very helpful to have an easy description of the hyperedges (or lines or subsets) of the hypergraph.

\subsection{Schematic Drawings}

Even if there is one "curved" line contained in the picture it does not really disturb the drawing as we can see in Fig. 1. The above mentioned result of Steinitz makes it reasonable to look for such drawings of configurations $v_{3}$ and to try to prove similar results for other classes of combinatorial structures.

If the number of "curved" lines is relatively small, such a picture is still much clearer than a picture using Venn diagrams.

\subsection{Nice Drawings}

Apart from the above topics the problem remains to find a nice linear drawing if there is one at all. Perhaps a nice drawing containing a few "curved" lines is more acceptable than an ugly linear drawing. 


\section{Other Combinatorial Structures}

The question of drawing hypergraphs is not restricted to configurations. They are just the best examples to introduce the topic because of their historical and geometric context. For many purposes in mathematics as well as in applications it is desirable to describe a certain structure by a drawing rather than by giving a list of points and lines.

\subsection{Linear Hypergraphs}

If the hypergraph is linear but not necessarily uniform or regular this does not change the problem discussed above very much. We can read through the above paragraphs and follow the same strategy again. In particular, it should be investigated which linear hypergraphs can be drawn with straight lines only or with at most "quadratic" line in the sense of Steinitz.

\section{$3.2(r, 1)$-Designs and Linear Spaces}

Perhaps the problem with linear hypergraphs is that although they are defined in books on hypergraphs so far they have not been investigated very much in detail.

A certain subclass, however, linear spaces and $(r, 1)$-designs have been enumerated for small numbers of points and are an interesting data base. In the language of hypergraphs a linear space is a hypergraph where through two given points there is always a common hyperedge. An $(r, 1)$-design is an $r$-uniform linear space.

Recently all small linear spaces and $(r, 1)$-designs have been constructed. For further information on all $(r, 1)$-designs with at most 13 points see [6], [7], and [9]. Moreover, all linear spaces with at most 11 points have been determined (see [12]).

\section{Conclusion}

In my opinion the strategy to draw hypergraphs or at least linear hypergraphs by displaying the hyperedges as lines (as far as possible ) will lead to drawings which in most cases will be better readable than traditional drawings. Moreover, this strategy is closely related to an interesting theoretical background in geometry, combinatorics, and algebra having its roots back in the $19^{\text {th }}$ century. For further details the reader has to be referred to the references below.

Of course, the drawing of hypergraphs is much more difficult than graph drawing. Perhaps this is the reason why so far it has not been attacked seriously. In particular, for lines of size greater than 3 the problem becomes very hard. For these hypergraphs only very few results have been obtained until now. I hope that this paper will start a more intensive research on the drawing of hypergraphs, from a theoretical and a practical point of view. 


\section{References}

1. C. Berge, Hypergraphs, Amsterdam-New York- Oxford-Tokyo (1989)

2. J. Bokowski, B. Sturmfels, Computational Synthetic Geometry, Springer LNM 1355, Berlin-Heidelberg-New York (1989)

3. G. DiBattista, P. Eades, H. de Fraysseix, P. Rosenstiehl, R. Tamassia (eds.), Graph Drawing '93 Proceedings, September 1993, Paris

4. H. Gropp, On the history of configurations, Internat. Symp. on Structures in Math. Theories, ed. A.Díez, J.Echeverría, A.Ibarra, Universidad dei País Vasco, Bilbao (1990) 263-268

5. H. Gropp, Configurations and graphs, Discrete Math. 111 (1993) 269-276

6. H. Gropp, Configurations and ( $r, 1)$-designs, Discrete Math. 129 (1994) 113-137

7. H. Gropp, Graph-like combinatorial structures in $(r, 1)$-designs, Discrete Math. 134 (1994) 65-73

8. H. Gropp, Configurations and their realization ( to appear)

9. H. Gropp, The $(r, 1)$-designs with 13 points ( submitted to Discrete Math. )

10. S. Kantor, Die Configurationen $(3,3)_{10}$, Sitzungsber. Akad. Wiss. Wien, math.naturwiss. Kl. 84 (1881) 1291-1314

11. J.G. Oxley, Matroid theory, Oxford-New York-Tokyo (1992)

12. C. Pietsch, On the classification of linear spaces of order 11, J. Combin. Des. 3 (1995), 185-193

13. E. Steinitz, Über die Construction der Configurationen $n_{3}$, Dissertation Breslau (1894)

14. V.I. Voloshin, On the upper chromatic number of a hypergraph, Australasian J. Comb. 11 (1995) 25-45

15. V.I. Voloshin, Hypergraph Drawing and Optimization System, preprint 